\title{
Untersuchung von Assoziationen zwischen dem Prion-Protein-Gen und Leistungseigenschaften beim Schaf an Stations- und Felddaten
}

\author{
Herrn Professor Dr. Dr. h.c. mult. Ernst Kalm zum 65. Geburtstag gewidmet
}

\begin{abstract}
Title of the paper: Association between prion - protein - haplotypes and yield traits of station and field tested sheep

On the basis of routinely collected data and genotypes of the prion Protein from stud book and performance testing of sheep in Thuringia, associations between prion - protein - haplotypes and traits of fattening, carcasses, fleece and fertility were estimated. The number of useable Animals was for different attributes (altogether, genotyped) fattening 7316/793, ultrasound 4733/793, carcass 3787/165, wool score 2198/795 and litter size 26000/1643. For survival- analysis were 20803 died animals, thereof 284 genotyped and 7257 living animals, thereof 1452 genotyped useable.

Most of these traits showed no association with haplotypes of the prion protein. For the breed "Merinolandschaf" there was a tendency towards finer wool for homozygous ARR-carriers. In general, beyond the effect of the reduced selection pressure, no disadvantage is expected for Thuringian breeds when ARR - Haplotypes proliferate. Survival analyses show that known ARR-carriers stay longer in the flock.
\end{abstract}

Key Words: prion - protein - haplotypes, Scrapie, fattening performance, carcass, wool, fertility, survival

\section{Zusammenfassung}

Anhand der in der Leistungsprüfung und Herdbuchführung für Schafe in Thüringen routinemäßig ermittelten Daten und Prion - Protein - Genotypen wurde nach Assoziationen zwischen den Prion - Protein - Haplotypen und Merkmalen der Mast-, Schlacht-, Woll- und Fruchtbarkeitsleistungen gesucht. Die Anzahl auswertbarer Tiere betrug für die einzelnen Merkmalskomplexe (insgesamt, genotypisiert): Mastleistung 7316/793, Ultraschall 4733/793 Schlachtleistung 3787/165, Wollbonitur 2198/795 und Wurfgröße 26000/1643. In einer Survival-Analyse waren 20803 abgegangene Tiere, davon 284 genotypisiert und 7257 lebende, davon 1452 genotypisiert auswertbar. Für die meisten Merkmale waren keine Assoziationen nachweisbar. Für Merinolandschafe deutet sich feinere Wolle für homozygote ARR-Träger an. Insgesamt wird kein Nachteil für die in Thüringen züchterisch bearbeiteten Rassen bei Vermehrung des ARR-Anteils erwartet, der über die Effekte des reduzierten Selektionsdruckes auf Leistungseigenschaften hinausgeht. Survival Analysen zeigen, dass schon jetzt bekannte homozygote ARR-Träger deutlich länger im Bestand verbleiben.

Schlüsselwörter: Prion-Protein-Haplotypen, Scrapie, Mastleistung, Schlachtkörper, Wolle, Fruchtbarkeit, Überleben

\section{Einleitung}

Eine Scrapie-Infektion stellt für Schafhalter und Tierseuchenkasse wegen der in der EU-Verordnung 999/2001 geforderten Keulung ein erhebliches wirtschaftliches Risiko dar.

Allerdings erkennt der Gesetzgeber das unterschiedliche Übertragungsrisiko in Abhängigkeit vom Prion-Protein-Genotyp an, so dass sich die Merzung auf Tiere mit höherem Infektionsrisiko beschränkt. Damit eröffnet sich für die Schafzucht die Mög- 
lichkeit zur Risikovorsorge, indem der Anteil bestimmter Genotypen in der Herde erhöht wird.

Dabei sind die Aminosäuren an den Positionen
136 Alanin (A) oder Valin (V)
154 Histidin $(\mathrm{H})$ oder Arginin (R) und
171(Arginin (R), Histidin (H) oder Glutamin (Q)

von Bedeutung. Bisher wurden die Haplotypen ARR, ARQ, AHQ ARH und VRQ als vorherrschend beschrieben. Übereinstimmend kommen alle bisher vorliegenden Studien zum Schluss, dass der Haplotyp ARR mit deutlich verbesserter Resistenz gegen die klassische Scrapie einhergeht und der Genotyp ARR/ARR nahezu vollständig resistent ist (ACIN et al., 2004; TONGUE et al., 2004; BAYLIS et al., 2004; BILLINIS et al., 2004 ; LÜHKEN et al., 2004).

In den wichtigen Thüringer Schafpopulationen bestehen gute Ausgangssituationen, um den Anteil ARR-Haplotypen in kurzer Zeit deutlich zu erhöhen. Insbesondere der ARR-Anteil der Merinolangwollschafe liegt deutlich über dem anderer Merinorassen (DRÖGEMÜLLER et al., 2001). Seit etwa zwei Jahren werden im Herdbuch bei den meisten Rassen nur noch Böcke mit mindestens einem ARR eingesetzt, weil Zuchtböcke ohne ARR auch in die Landeszucht kaum noch, und wenn, dann zu deutlich schlechteren Preisen, absetzbar sind.

Insofern stellt sich die Frage, ob Assoziationen zwischen Scrapie - Typen und Leistungsmerkmalen bestehen. Bisherige Studien (PROKOPOVA et al., 2002; DE VRIES et al., 2003, 2004a,b,c; BRANDT et al., 2004) lassen keine Beziehungen erwarten. Andererseits besteht die dringende Notwendigkeit, dieser Frage auch an diesem Material nachzugehen, bevor die Selektion auf Scrapieresistenz zu unerwünschten korrelierten Selektionserfolgen in den Leistungsmerkmalen führt. Schon nach drei Jahren mit umfangreicher Untersuchung und Selektion der Prion - Protein Genotypen wird die Zuchtleitung mit der Behauptung konfrontiert, ARR-tragende Tiere seien weniger vital.

Ziel dieser Untersuchungen ist, die Frage $\mathrm{zu}$ klären, ob in den größeren Rassebeständen Thüringens Assoziationen zwischen den Prion - Protein - Genotypen und den Komplexen Mastleistung, Schlachtkörperqualität, Wolle, Fruchtbarkeit und Lebensdauer vorliegen.

\section{Material und Methoden}

Prion - Protein - Bestimmung

Bis auf wenige Ausnahmen wurden alle EDTA - Blutproben durch den Schafgesundheitsdienst des TGD Thüringen e.V. entnommen. Bei der Probennahme wird durch regionale Zuchtberater die Dokumentation und deren Plausibilität geprüft. Alle weiteren Arbeiten zur Genotypenbestimmung erfolgten in verschiedenen, mittels Ringtest zertifizierten, deutschen Laboren.

\section{Mast- und Schlachtleistung}

Das Material zur Bewertung der Mast- und Schlachtleistung entstammt der Datenbank zur Zuchtwertschätzung. Folgende Merkmale wurden ausgewertet:

Zunahme in der Aufzucht bis zur Anlieferung auf Station ZA (g/Tag)

Prüftagszunahme PTZ (g/Tag) 
Ultraschall Kotelettmuskeldicke USK (mm)

Ultraschall Rückenfettdicke USF (mm)

Schlachtkörperbonitur Blatt BL (1-9)

Schlachtkörperbonitur Rücken $\mathbf{R}$ (1-9)

Schlachtkörperbonitur Keule K (1-9)

Schlachtkörperbonitur Oberflächenfett OF (1-9)

Nierenfettmenge NF (g).

Die detaillierte Beschreibung der zu Grunde liegenden Prüfung findet sich bei GERNAND und LENZ (2005). Eine Übersicht über die insgesamt geprüften Tiere enthält Tabelle 1.

Tabelle 1

Tierzahl (N) für die PrP-Untersuchung, die Zunahme, die Ultraschallmessung, den Schlachtkörperwert und die Wollmerkmale nach Rassen (Number of animals (N) for PrP-Analysis, gain, ultrasound measurement, carcass value and wool traits across breeds)

\begin{tabular}{llrrrr}
\hline Rasse & \multicolumn{2}{c}{ PrP } & & & \\
& Untersuchung & Zunahme & Ultraschall & Schlachtkörper & Wolle \\
& & $\mathrm{N}$ & $\mathrm{N}$ & $\mathrm{N}$ & 221 \\
\hline Merinoland & nein & 389 & 334 & 278 \\
(ML) & ja & 94 & 93 & 94 \\
Merinolangwoll & nein & 4691 & 2865 & 2595 & 1368 \\
(MLW) & ja & 432 & 434 & 61 & 434 \\
Schwarzköpfiges & nein & 162 & 138 & 86 & 95 \\
Fleischschaf (SKF) & ja & 59 & 59 & 11 & 59 \\
Leineschaf & nein & 386 & 365 & 314 & 263 \\
(LEI) & ja & 145 & 144 & 63 & 145 \\
Suffolk & nein & 127 & 100 & 48 & 47 \\
(SU) & ja & 33 & 33 & 10 & 33 \\
& nein & 768 & 138 & 358 & 45 \\
Sonstige & ja & 30 & 30 & 2 & 30 \\
\hline
\end{tabular}

Im ersten Schritt wurde die Erweiterung der Modelle aus der Routine-Zuchtwertschätzung (GERNAND und LENZ, 2005) um die Prion - Protein - Typen mittels GLM Prozedur univariat (SPSS12.0) geprüft. Dabei wurden der Vater anstelle des Tiereffektes als zufälliger Effekt und die Häufigkeit der verschiedenen Haplotypen (unbestimmt,0,1,2) und ihre Interaktionen mit der Rasse beachtet. Die durchgängig nicht signifikanten Interaktionen für Mast- und Schlachtleistungsmerkmale wurden schrittweise aus dem Modell entfernt. Da keine der Allelhäufigkeiten in einem der Mast- und Schlachtleistungsmerkmale eine Irrtumswahrscheinlichkeit von weniger als $10 \%$ erreichte, erfolgte auch deren Entfernung - mit Ausnahme von ARR - aus dem Modell.

Die Besten linearen unverzerrten Schätzer (BLUE) der ARR Kombinationen [ohne ARR (XXX / XXX, ein ARR, kombiniert mit einen beliebigen anderen Haplotyp (ARR / XXX) und ARR/ARR] zur Masse der unbestimmten wurden mittels des Programm-Paketes PEST (GROENEVELD et al., 1993) geschätzt. Die Modelle und die Varianz - Covarianz - Matrix entsprachen denen der Routine-Zuchtwertschätzung (GERNAND und LENZ, 2005).

Wolle

Für die Untersuchung der Wolleigenschaften fanden das gleiche Material und die gleiche Vorgehensweise wie bei der Mast- und Schlachtleistung Anwendung. Die Daten 
entstammen der auf der Prüfstation vorgenommenen Wollbonitur im Alter von ca. 4 Monaten. Die neben dem Vater, den Scrapie - Allelen und ihren Interaktionen mit der Rasse im Modell aufgenommen Faktoren sind Tabelle 2 zu entnehmen.

Tabelle 2

Einflussfaktoren auf die Wolleigenschaften (Factors influencing fleece characteristics)

\begin{tabular}{lccccc}
\hline & Boniturdatum & Rasse & Herkunftsbetrieb & $\begin{array}{c}\text { Körpermasse } \\
\text { (Covariable) }\end{array}$ & Geburtstyp \\
\hline Stapelhöhe & + & + & + & + & \\
Wollfeinheit & + & + & + & & + \\
Wollqualität & + & + & + & + & \\
\hline
\end{tabular}

Im Gegensatz zu den Mast- und Schlachtleistungsmerkmalen erreichte die Interaktion der ARR -Kombinationen (unbestimmt, ohne ARR, ein ARR, kombiniert mit einem beliebigen anderen Haplotyp und ARR/ARR) * Rasse für die Wollfeinheit zumindest den Grenzbereich (5,4 \% Irrtumswahrscheinlichkeit) der Signifikanz. Die Häufigkeiten anderer Allele blieben dagegen ebenso wie ihre Interaktionen auch für Wolle ohne Bedeutung. Die Schätzung der Interaktionen Rasse * ARR Klassen erfolgte ebenfalls mit dem Modell aus der Routinezuchtwertschätzung, wobei der Rasseeffekt durch die Interaktion Rasse * ARR - Klassen ersetzt wurde.

\section{Fruchtbarkeit}

Die Tabelle 3 zeigt das verfügbare Datenmaterial aus der Herdbuchführung des Landesverbandes Thüringer Schafzüchter e. V.

Tabelle 3

Anzahl Lammungen nach ARR - Typen und Rassen (Number of lambings across ARR - genotype and breed)

\begin{tabular}{lrrrr}
\hline & \multicolumn{3}{c}{ Anzahl ARR } \\
\hline ML & 0 & 1 & 2 & unbestimmt \\
MLW & 174 & 77 & 10 & 7701 \\
SKF & 729 & 738 & 154 & 64242 \\
SU & 5 & 12 & 48 & 3349 \\
LEI & 39 & 337 & 213 & 1668 \\
RHO* & 8 & 21 & 21 & 2603 \\
Sonstige & 21 & 45 & 41 & 6098 \\
Alle & 35 & 38 & 16 & 1449 \\
\hline
\end{tabular}

*Rhönschaf

Das Material umfasst etwa 90000 Lammungen von 26000 Muttern. Davon waren 1643 Schafe untersucht, von denen insgesamt 2782 Lammungen im Material vorlagen. Verwendet wurde die Anzahl lebend geborener Lämmer. Da es sich bei der Anzahl lebend geborener Lämmer um ein rangkategoriales Merkmal handelt, wurde mittels einer ordinalen Regression (Prozedur PLUM - Polytomus Universal Model) ausgewertet.

Das dabei gewählte kumulative Logit-Modell erklärt für ein Kriterium mit J Stufen die jeweilige kumulative Wahrscheinlichkeit $P(Y \leq J \mid X)$ für $j=1,2$...J-1. 


$$
P(Y \leq \mid X)=\frac{e^{\alpha_{j}-\beta X}}{1+e^{\alpha_{j}-\beta X}} \text { mit } \beta:=\left[\beta_{1}, \beta_{2 \ldots} \beta_{M}\right] \text { und } X:=\left[\begin{array}{c}
X_{1} \\
X_{2} \\
\vdots \\
X_{M}
\end{array}\right], J=1,2, \ldots J-1
$$

Gegensätzlich zum binären Modell wird die Wahrscheinlichkeit der Zugehörigkeit zu einer Kategorie hier mit dem Index kleiner oder gleich J modelliert. Um der intuitiven Erwartung zu entsprechen, dass Schätzer mit positiver Kriteriumskorrelation auch ein positives Vorzeichen aufweisen, werden in der SPSS - Prozedur PLUM die Regressionsparameter mit einem negativen Vorzeichen versehen. Die SAS-Prozedur LOGISTIC verzichtet hierauf und liefert betragsgleiche Ergebnisse mit umgekehrten Vorzeichen.

Für die Interpretation der Schätzwerte ist die logarithmierte Darstellung hilfreich:

$$
\ln \left(\frac{P(y \leq j \mid X)}{P(y>j \mid X)}\right)=\alpha_{j}-\beta X, j=1,2, . ., J-1
$$

Der Parameterschätzer von $\exists$ beschreibt die erwartete Änderung des logarithmierten Verhältnisses der Wahrscheinlichkeiten.

Mit der Masse an unbestimmten Tieren werden die Tiere ohne ARR, mit einem ARR, und mit 2 ARR - Allelen verglichen.

Da es kaum Verknüpfungen zwischen den Rassen über die Betriebe gab und möglicherweise die saisonalen Effekte bei den verschiedenen Rassen unterschiedlich ausgeprägt waren, erfolgten mehrere Rechenläufe für jede der häufigeren Rassen extra und einmal über alle Rassen mit Rasse als Einflussfaktor.

Die Scrapie - Ergebnisse weiblicher Tiere basieren fast durchgängig auf der Untersuchung von Zutretern in den letzten drei Jahren. Deshalb war hier der Anteil Erstlingslammungen viel höher als im Gesamtmaterial. Somit musste das Alter des Schafes unbedingt beachtet werden. Daneben hatten auch Rasse, Betrieb * Jahr und der Deckmonat einen wesentlichen Einfluss auf die Anzahl Lämmer und mussten im Modell beachtet werden. Zusätzlich ging der Vater des Mutterschafes in das Modell ein.

\section{Überlebensrate}

Die Daten hierzu entstammten ebenfalls den Herdbuchdateien des Landesverbandes Thüringer Schafzüchter e. V. Allerdings war der tatsächliche Abgangstermin bei ca. 30\% der Abgänge nicht verifizierbar, da ein plausibles Abgangsdatum nicht immer verfügbar ist. Deshalb wurde mit dem gröberen Abgangsjahr gearbeitet. Dabei wurde auch das Jahr der letzten Lammung als Abgangsjahr benutzt, wenn die Lammung vor 2003 stattfand und im Datenmaterial kein späterer Eintrag, der Rückschlüsse auf den Verbleib in der Herde zuließ, zu finden war. Aus der Differenz zwischen dem letzten Lammjahr und dem Geburtsjahr wurde das Lebensalter in Jahren ermittelt. Deshalb konnten nur Schafe ausgewertet werden, von denen mindestens eine Lammung eingetragen war.

Tabelle 4 zeigt die Altersverteilung aller im Herdbuch eingetragenen Schafe. Da die Untersuchungen der Prion - Protein - Gene erst in den letzten Jahren ausgebaut wur- 
den, kann die Mehrheit der untersuchten Tiere maximal das dritte Lebensjahr erreicht haben.

Tabelle 4

Häufigkeiten (N) der Herdbuchschafe nach Altersklassen (Frequencys (N) of stud book sheep for age classes)

\begin{tabular}{crrrr}
\hline & \multicolumn{2}{c}{ Nicht untersucht } & Untersucht \\
Altersklasse (Jahre) & lebend $(\mathrm{N})$ & tot $(\mathrm{N})$ & lebend $(\mathrm{N})$ & tot $(\mathrm{N})$ \\
\hline 0 & 21 & 9 & 24 & 3 \\
1 & 98 & 973 & 388 & 86 \\
2 & 716 & 2565 & 695 & 86 \\
3 & 1206 & 3156 & 248 & 16 \\
4 & 1303 & 3356 & 70 & 7 \\
5 & 975 & 3150 & 15 & 18 \\
6 & 778 & 2985 & 10 & 15 \\
7 & 390 & 2354 & 1 & 8 \\
8 & 225 & 1247 & & 6 \\
9 & 59 & 530 & 1 & 3 \\
10 & 32 & 186 & & \\
11 & 1 & 32 & & \\
12 & 1 & 6 & & \\
\hline
\end{tabular}

Zur Anwendung kam die im SPSS 12.0 implementierte Survival - Analyse mit CoxRegression.

Aufgrund der unterschiedlichen Ausgangssituation ist die Bedeutung der Prion - Protein - Typen in den einzelnen Rassen sehr unterschiedlich. Deshalb wurden die wichtigen Rassen getrennt ausgewertet.

Das Modell berücksichtigt neben den Prion - Protein - Typen den Faktor Betrieb * Geburtsjahr. Dabei wurden alle Haplotypen außer ARR und VRQ zu sonstigen (XXX) zusammengefasst. Saisonal unterschiedliche Abgangsrisiken können aufgrund des ungenauen Abgangszeitpunktes nicht beachtet werden.

\section{Ergebnisse}

Mast und Schlachtleistung

In fast keinem Fall sind die in Abbildung 1 dargestellten Kontraste zwischen den identifizierten Prion - Protein - Genotypen für die Mast- und Schlachtleistungsmerkmale signifikant. Nur der Kontrast der Prüftagszunahme zwischen den unbestimmten und den Tieren, die nachweislich kein ARR tragen, überschreitet die Signifikanzgrenze. Dies deutet auf eine gezielte Auswahl der untersuchten Tiere. Für Bocklämmer, die keine Aussicht auf züchterischen Einsatz haben, wurden die Aufwendungen gespart. 

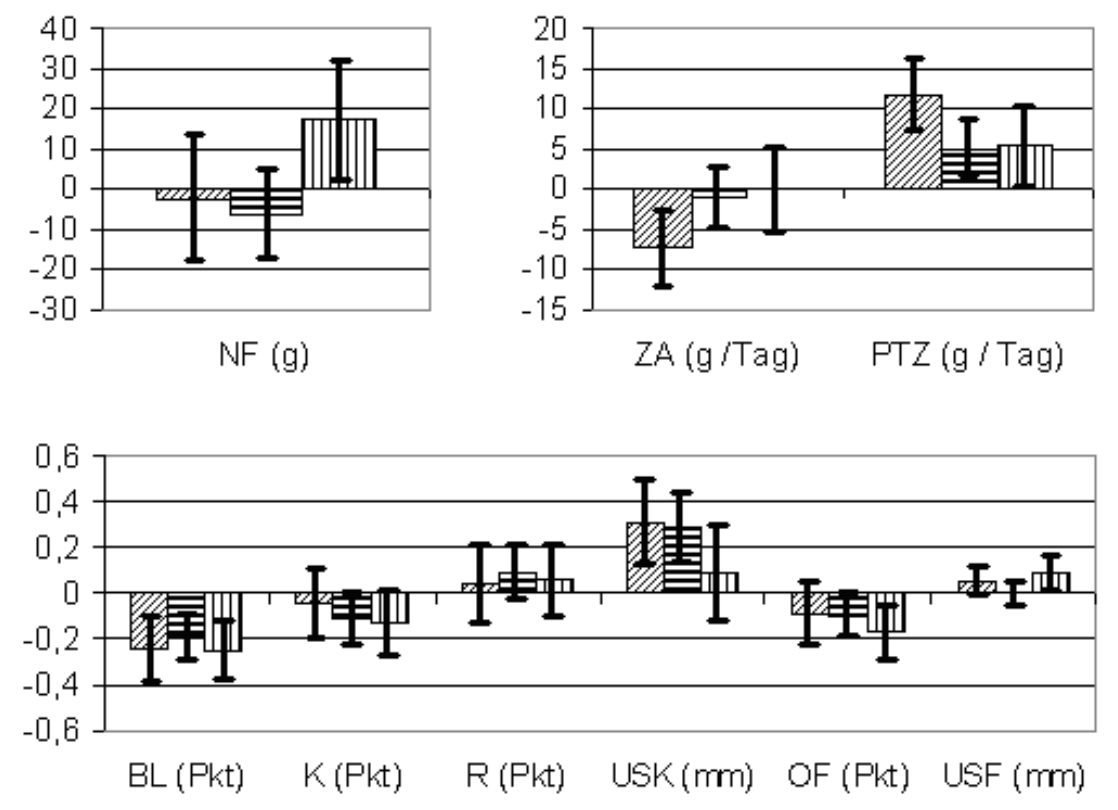

四XXX/XXX $=\mathrm{ARR} / \times \times \times$ 四ARR/ARR

Abb. 1: BLUE - Schätzer [und Standardfehler] für Merkmale der Mast- und Schlachtleistung verschiedener Prion - Protein - Genotypen [unbestimmte $=0$ ] (BLU-Estimates [and standard errors] of fattening and carcass traits for different prion - protein - genotypes $[$ unknown $=0]$ )

Wolle

Von den Wollmerkmalen zeigt lediglich die Interaktion ARR * Rasse für die Wollfeinheit einen im Grenzbereich (Irrtumswahrscheinlichkeit 5,4) signifikanten Effekt.

Für die Wollmerkmale wurden deshalb die Effekte von Rasse * Scrapie - Typ geschätzt (Abb. 2-4).

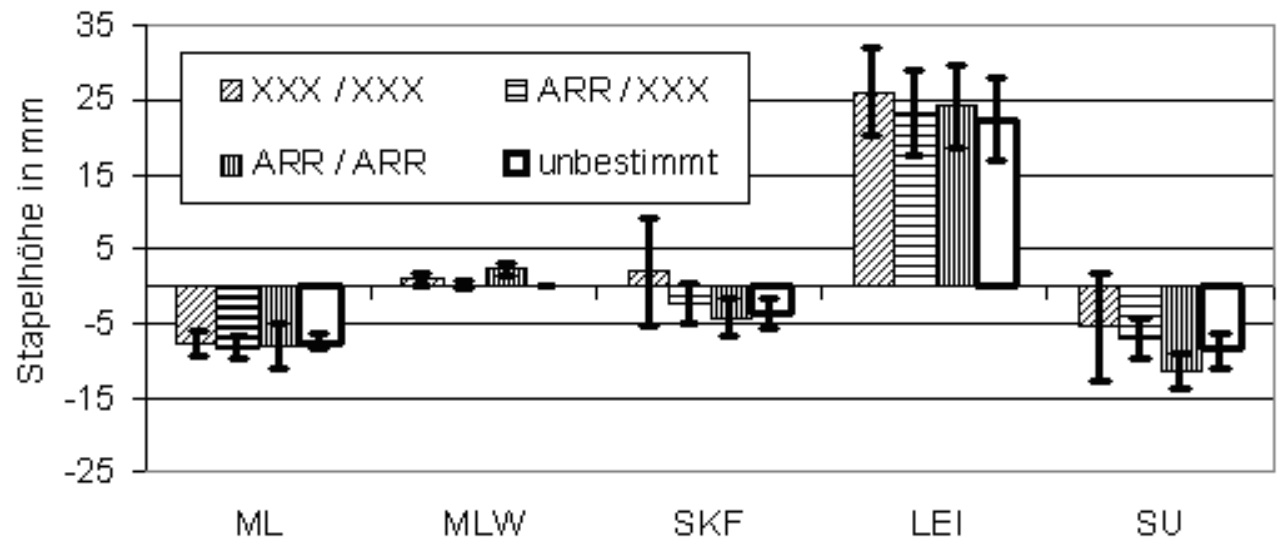

Abb. 2: BLUE - Schätzer [und Standardfehler] der Stapelhöhe für die Interaktion Prion - Protein - Genotyp * Rasse [unbestimmte MLW=0] (BLU-Estimates [and standard errors] of staple length for the interaction prion protein - genotyp *breeds [unknown MLW=0])

Allerdings ist die Interaktion für die Wollfeinheit (Abb. 3) nur durch einige wenige Merinoland-Schafe mit ARR/ARR verursacht, die wesentlich feinere Wolle haben als die anderen Merinoland-Schafe. In den anderen Rassen können keine Effekte vermutet werden. Für die Stapelhöhe (Abb. 2) und die Wollqualitätsnote (Abb. 4) sind keine Einflüsse zu erkennen. 


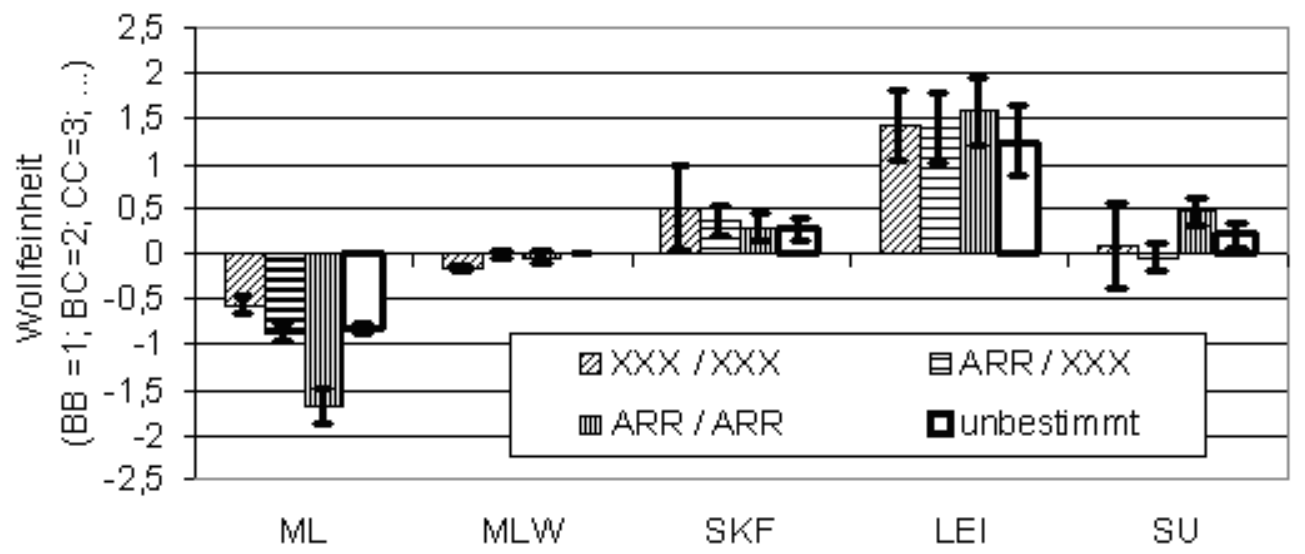

Abb. 3: BLUE - Schätzer [und Standardfehler] der Wollfeinheit für die Interaktion Prion - Protein - Genotyp * Rasse [unbestimmte MLW=0] (BLU-Estimates [and standard errors] of wool fineness for the interaction prion protein - genotyp *breeds [unknown MLW=0])

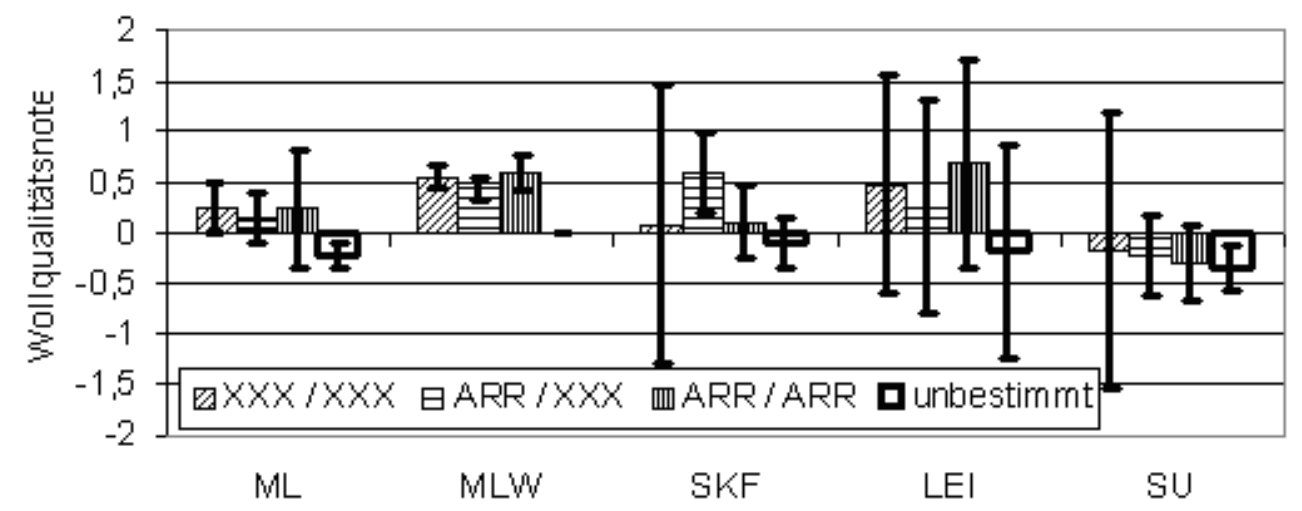

Abb. 4: BLUE - Schätzer [und Standardfehler] der Wollqualität für die Interaktion Prion - Protein - Genotyp * Rasse [unbestimmte MLW=0] (BLU-Estimates [and standard errors] of wool quality score for the interaction prion - protein - genotyp *breeds [unknown MLW=0])

Tabelle 5

Ordinale Regressionsparameter der -Prion - Protein -Genotypen nach Rassen (Ordinal regression between prion - protein - genotypes for breeds)

\begin{tabular}{lcccccc}
\hline & \multicolumn{2}{c}{ XXX / XXX } & \multicolumn{2}{c}{ ARR / XXX } & \multicolumn{2}{c}{ ARR / ARR } \\
& $\mathrm{B}$ & $\mathrm{S}_{\mathrm{B}}$ & $\mathrm{B}$ & $\mathrm{S}_{\mathrm{B}}$ & $\mathrm{B}$ & $\mathrm{S}_{\mathrm{B}}$ \\
\hline Alle Rassen & $-0,050$ & 0,088 & $-0,005$ & 0,083 & $-0,020$ & 0,123 \\
ML & 0,290 & 0,215 & 0,458 & 0,265 & $-0,482$ & 0,710 \\
MLW & $-0,117$ & 0,107 & $-0,095$ & 0,108 & $-0,256$ & 0,199 \\
SKF & 0,122 & 1,032 & $-1,010$ & 0,634 & $-0,467$ & 0,419 \\
LEI & $-0,782$ & 0,836 & $-0,154$ & 0,487 & 0,662 & 0,523 \\
SU & $-0,532$ & 0,429 & 0,148 & 0,190 & 0,270 & 0,223 \\
RHO & 1,113 & 0,708 & 0,266 & 0,516 & 0,118 & 0,529 \\
\hline
\end{tabular}

\section{Fruchtbarkeit}

Tabelle 5 zeigt die mittels ordinaler Regression geschätzten Parameter für die Fruchtbarkeit der untersuchten und nach dem ARR - Vorkommen eingeteilten Gruppen gegenüber der großen Masse unbestimmter Tiere über alle Rassen und innerhalb der Rassen. Die Schätzwerte für die einzelnen Rassen schwanken zum Teil, weil sie bei 
den selteneren Rassen und Genotypen nur unsicher ermittelt werden können. Sie sind aber in keinem Fall signifikant.

Die über die Rassen erzielten Ergebnisse liegen dagegen nahe Null, so dass sich kaum Veränderungen in der Mehrlingsrate zur Ausgangsfrequenz der unbestimmten ergeben. Insgesamt ist also davon auszugehen, dass die angestrebte Erhöhung des ARR Anteils in den Populationen zu keinen erkennbaren Veränderungen in der Mehrlingshäufigkeit führen wird!

\section{Überlebensrate}

Die Survival - Analyse konvergierte nur für die Merinolangwollschafe. Die unterschiedlichen Überlebenskurven für Merinolangwollschafe können der Abbildung 5 entnommen werden.

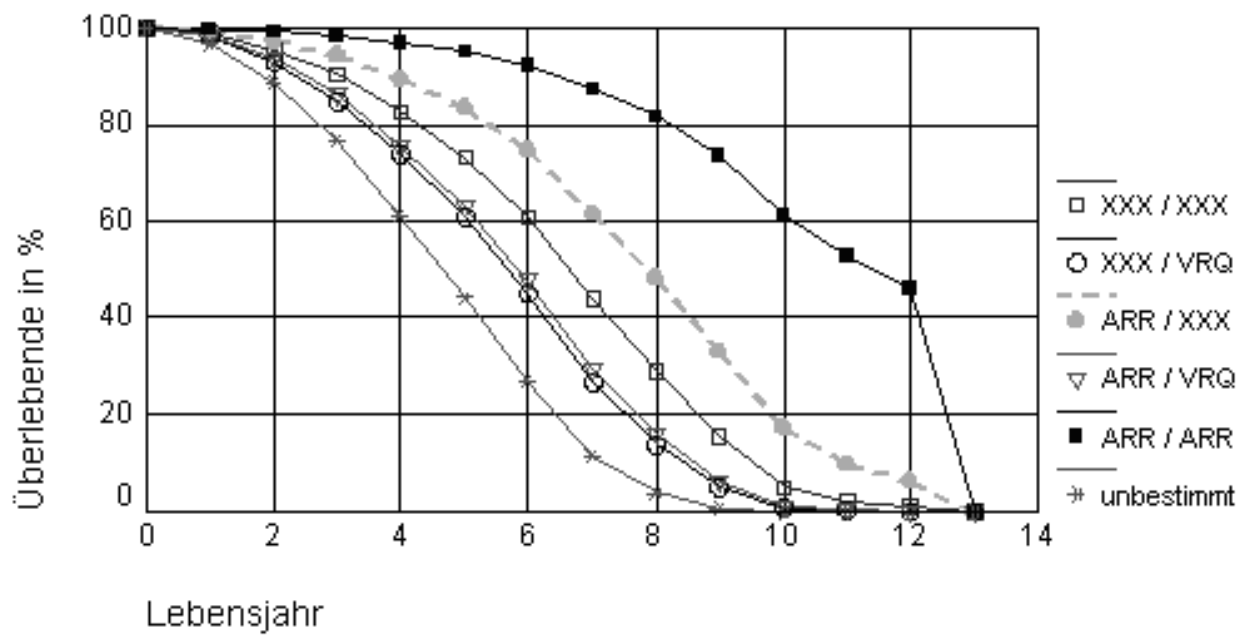

Abb. 5: Kumulative Überlebenskurven nach Prion - Protein - Genotypen (Cumulative survival curves for prion protein types)

Es ist zu erkennen, dass Tiere mit Prion - Protein - Bestimmung unabhängig vom Typ länger in der Zucht verbleiben als ohne. Bekannte ARR/ARR - Genotypen weisen dabei erwartungsgemäß die mit Abstand höchsten Überlebensraten auf, Träger eines ARR - Haplotypes ohne VRQ nehmen eine Zwischenstellung ein. Im Gegenzug führt VRQ zu einer früheren Merzung, auch wenn es mit ARR kombiniert auftritt.

\section{Diskussion}

Für die Mast- und Schlachtleistung ist in keinem Fall der Effekt eines Scrapie - Allels oder einer Interaktion mit einem Scrapie - Allel signifikant. Die Ergebnisse hier legen ebenso wie die von DE VRIES et al. (2003, 2004a,b), BRANDSMA et al. (2004) und BRANDT et al. (2004) nahe, dass keine Assoziationen zwischen Prion - Protein - Genotyp und Mast- oder Schlachtleistung existieren. Aber auch bei der Suche nach QTL wird Chromosom 13, wo die Prion - Proteine codiert werden, nicht zu den Kandidatenregionen gezählt (WALLING et al., 2004). Insgesamt ist davon auszugehen, dass die angestrebte Erhöhung des ARR - Anteils in der Population lediglich über die Einschränkung des Selektionsdruckes auf die Merkmale der Mast- und Schlachtleistung zu einer vorübergehenden Beeinträchtigung des Zuchtfortschrittes führen wird. 
Damit verbunden wird das Entstehen einer vorübergehenden Assoziation zwischen Prionen - Allelen und Leistung. Abbildung 6 macht deutlich, dass untersuchte Jungböcke ohne ARR nur mit extrem gutem Zuchtwert für die Nachkommenerzeugung ausgewählt werden. Deshalb weisen die nachkommengeprüften Böcke ohne ARR eine sehr niedrige Frequenz und trotz ihrer niedrigen Anzahl signifikant bessere Zuchtwerte auf. Diese Situation ist typisch für das Auftauchen einer Verknüpfung von Genotyp und Leistung, ohne dass eine genetische Kopplung zwischen beiden besteht. Sie ist selektiv bedingt und entsteht in der Phase der Umzüchtung. Hierauf sollten die Zuchtleitung und die Züchter eingestellt sein. Nur weil das Datenmaterial aus dem Beginn der Erhebung von Prion - Protein - Genotypen stammt, sind die entsprechenden Effekte unterschiedlicher Vorselektion der verschiedenen Genotypen noch nicht gravierend. Auch ein genereller Vorteil der genotypisierten gegenüber den nicht untersuchten Tieren wie bei DE VRIES et al. (2004a,b) ist hier nicht durchgängig zu erkennen. Lediglich die Überlegenheit der Tiere ohne ARR über die unbestimmten in der Prüftageszunahme (Abb. 1) deutet auf einen solchen Effekt. Jungböcke mit guten Zunahmen oder mit einer Abstammung, die ein ARR - Alleel erhoffen läßt, wurden für die Genotypenbestimmung zu höheren Anteilen ausgewählt.

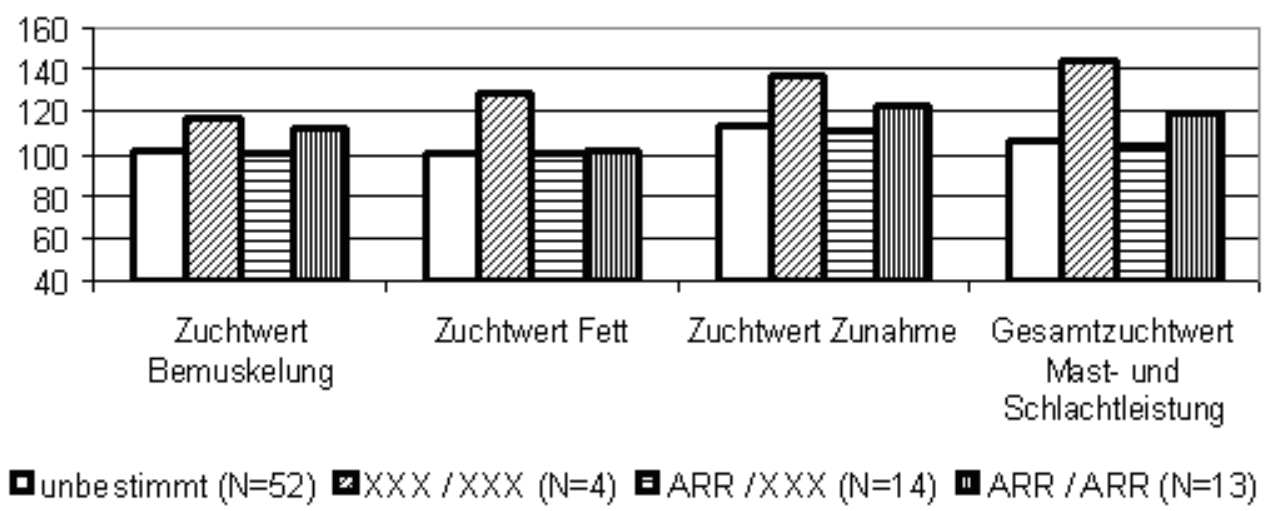

Abb. 6: Zuchtwerte von Böcken mit mindesten 8 auf Station geprüften Nachkommen (Breeding values of rams with a minimum of 8 station tested progenies)

Abgesehen von der Wollfeinheit der Merinolandschafe waren für Wollmerkmale ebenso wie bei DE VRIES et al. (2004a,b) keine signifikanten Assoziationen zu beobachten. Bei der Wollfeinheit deuten sich Effekte der Scrapie - Genotypen im Grenzbereich zur Signifikanz an. Sie entstehen, weil die wenigen Merinolandschafe mit ARR/ ARR deutlich feinere Wolle aufweisen als die anderen Gruppen. Insofern muss die Aussage hier unsicher bleiben.

Insgesamt bleiben die Schlussfolgerungen zur Wolle im Gegensatz zu den anderen Merkmalskomplexen etwas unsicherer. Ob die Wolle bei Merinolandschafen mit der Zucht auf Scrapieresistenz tatsächlich feiner wird, bleibt fraglich, sollte aber wegen der möglichen Bedeutung der Wollfeinheit für die Stabilität gegen hohe Niederschlagsmengen und damit die Landschaftspflegeeignung an anderer Stelle geprüft werden. Möglicherweise sind tatsächlich Gene mit Auswirkungen auf Wollmerkmale in der Nähe des Prionen - Gene zu finden, die vielleicht nur in einigen Rassen heterozygot sind. Ebenso könnten genetische Ungleichgewichte, wie sie auch zwischen ungekoppelten Genen beobachtet wurden (FARNIR et al., 2000; MC RAE et al., 2002) als Ursache der Befunde nicht auszuschließen sein. 
Die Ergebnisse zur Fruchtbarkeit lassen ebenso wie die Untersuchungen von BRANDT et al. (2004), BRANDSMA et al. (2004) oder DE VRIES (2004c) nicht erwarten, dass eine Erhöhung der ARR - Frequenz zu einer veränderten Mehrlingshäufigkeit führt.

Die Wahrscheinlichkeit, ein Tier im Folgejahr als lebend im Material wiederzufinden, steigt mit der Genotypisierung. Selbst VRQ - Träger finden sich häufiger wieder als der Durchschnitt nicht genotypisierter Schafe. Verantwortlich hierfür ist möglicherweise die mit der Genotypisierung erzwungene größere Sorgfalt bei der Kennzeichnung und Datenerfassung.

Unterschiedliche Überlebensraten zwischen den Genotypen sind im Herdbuch aufgrund der unterschiedlichen züchterischen Wertigkeit zu erwarten.

ARR/ARR - Tiere werden in den ersten Lebensjahren kaum gemerzt. Die extrem niedrige Abgangsrate in den ersten Lebensjahren lässt vermuten, dass hier nur absolute Zwangsabgänge hingenommen werden. ARR - Tiere verbleiben vermutlich auch mit Gesundheitsproblemen im Bestand. So sind im 4. Lebensjahr von den ARR/ARR-Tieren etwa 98\% im Bestand verblieben, während von den VRQ tragenden und den ungeprüften fast $40 \%$ bereits abgegangen sind. Zwangsläufig werden die Züchter mit Gesundheitsproblemen dieser Genotypen - Gruppe verstärkt konfrontiert und kurieren diese wegen des besonderen Wertes dieser Zuchttiere intensiver. Die hier vorgestellten Zahlen sollten in der Diskussion genutzt werden, um der unter Praktikern bereits entstandenen, aber falschen Auffassung, dass ARR - Tiere weniger vital seien, entgegenzutreten.

\section{Fazit}

Die Ergebnisse zeigen, dass die Scrapie - Resistenzzucht bereits intensiv betrieben wird. Assoziationen zwischen Scrapie - Typen und Leistungseigenschaften sind allerdings kaum zu beobachten. Auch wenn im Einzelnen die Power in einige Rassen und Untersuchungen auf Grund der geringen Stichprobenzahl nicht immer für sichere Schlussfolgerungen ausreichend ist, legt die Übereinstimmung der vorliegenden Untersuchungen die Schlussfolgerung nahe, dass keine wesentlichen Assoziationen zwischen Prion-Proteinen und Leistungseigenschaften vorliegen. Gelegentlich gefundene Beziehungen wie hier für Wollfeinheit sollten dabei nicht überbewertet werden. Sie werden im Regelfall an anderen Materialien nicht bestätigt. Untersuchungen zu tatsächlichen genetischen Kopplungen sind aufwendiger und verlangen umfangreicheres Datenmaterial mit informativen Erbgängen. Da die a priori-Wahrscheinlichkeiten von Kopplungen zwischen Prion-Protein-Haplotypen zu QTL sehr niedrig sind und keine physiologischen Zusammenhänge bekannt sind, die Pleiotropie vermuten lassen könnten, kann das Fehlen von Kopplungsanalysen kein Grund sein, die von den Züchtern betriebene Erhöhung des ARR-Anteils in der Population zu bremsen, wenn die Ausgangsfrequenzen keinen zu engen Flaschenhals befürchten lassen.

$\mathrm{Zu}$ beachten ist aber in der Zuchtpraxis, dass in der Umzüchtungsphase Böcke ohne ARR nur ausgewählt werden, wenn sie in anderen Merkmalen für besonders wertvoll gehalten werden. Die für die verschiedenen Genotypen ungleichen Selektionsintensitäten werden aber zu unterschiedlichen Leistungen der verschiedenen Genotypen führen. Ähnliche Resultate wird es durch die geringe Merzung weiblicher ARR - Träger auch hinsichtlich Exterieur- und Fitnessmerkmalen geben. Solche Effekte sind nicht durch eine genetische Kopplung, sondern allein durch unterschiedlich starke 
Leistungsselektion verschiedener ARR - Typen begründet. Hierauf sollten Zuchtleitung und Züchter eingestellt sein.

\section{Literatur}

ACIN, C.; MARTIN-BURRIEL, I.; GOLDMAN, W.; LYAHYAI, J.; MONZON, M.; R. BOLEA, R.; SMITH, A.; RODELLAR, C.; BADIOLA, J.J.; ZARAGOZA, P.:

Prion protein polymorphisms in healthy and scrapie-affected Spanish sheep. J. Gen. Virol. 85 (2004), $2103-2110$

BAYLIS, M.; CHIHOTA, C.; STEVENSON, E.; GOLDMAN, W.; SMITH, A.; SIVAM, K.; TONGUE, S.; GRAVENOR, M.B.:

Risk of scrapie in British sheep of different prion protein genotype. J. Gen. Virol. 85 (2004), 2735 2740

BILLINIS, C.; PSYCHAS, V.; LEONIDES, L.; SPYROU, V.; ARGYROUDIS, S.; VLEMMAS, I.; LEONTIDES, S.; SKLAVIADIS, T.; PAPADOPOULOS, O:

Prion protein gene polymorphisms in healthy and scrapie - affected sheep in Greece. J. Gen. Virol. 85 (2004), 547 - 554

BRANDSMA, J.H.; JANSS, L.L.G.; VISSCHER, A.H.:

Associations between PrP genotypes and litter size and 135 days weight in Texel Sheep. Livest. Prod. Sci. 85 (2004), 59-64

BRANDT, H.; LÜHKEN, G.; LIPSKY, S.; ERHARD, G.:

Der Einfluss von Polymorphismen im Prionprotein - Gen bei Merinoland- und Rhönschafen auf Fruchtbarkeitsmerkmale bei Mutterschafen und Gewichte bei Lämmern. Züchtungskunde 76 (2004), $344-353$

DE VRIES, F.; HAMANN, H.; DROGEMÜLLER, C.; ANRZEJEWSKI, M.; GANTHER, M.; DISTL, O.:

Einfluss der Prion-Protein Gen Polymorphismen auf Leistungsmerkmale bei deutschen Fleischschafrassen. Dtsch. Tierärztl. Wschr. 111 (2004b), 349-354

DE VRIES, F.; HAMANN, H.; DROGEMÜLLER, C.; GANTHER, M.; DISTL, O.:

Analysis of Associations between the prion protein genotype and reproduction traits in meat sheep breeds. Anim. Sci. 79 (2004c), 397-404

DE VRIES, F.; BORCHERS, N.; HAMANN, H.; DROGEMÜLLER, C.; REINICKE, S.; LÜPPING, W.; DISTL, O.:

Associations between the prion protein genotype and performance traits of meat breeds of sheep. Vet. Rec. 155 (2004a), 1401-143

DE VRIES, F.; BORCHERS, N.; DROGEMÜLLER, C.; HAMANN, H.; REINICKE, S.; LÜPPING, W.; DISTL, O.:

Analyse der Assoziation zwischen den Prionproteingenotypen und Leistungsmerkmalen bei Fleischschafrassen. Züchtungskunde 75 (2003), 249 - 258

DRÖGEMÜLLER, C.;. LEEB, T.; DISTL, O.:

Breeding for resistance to scrapie: Detection of polymorphisms in the prion protein gen in German sheep breeds. Arch. Tierz., Dummerstorf 44 (2001) Special Issue, 280-287

FARNIR, F.; COPPETIERS, W.; ARRANZ, J.J.; BERZI, P.; CAMBISANO, N.: GRISART, B.; KARIM, L.;

MARCQ, F.; MOREAU, L.; MNI, M.; NEZER, C.; SIMON, P.; VANMANSHOVEN, P.; WAGENAAR, D.;

GEORGES, M.:

Extensive genome-wide linkage disequilibrium in cattle. Genome Res. 10 (2000), 220-227

GERNAND, E.; LENZ, H.:

Nutzung des Ultraschalls in der Schlachtleistungsprüfung und Zuchtwertschätzung für Schafe. Arch. Tierz., Dummerstorf 48 (2005) 2, 174-184

GROENEVELD, E.; KOVAC, M.; WANG, T.:

Pest Version 3.1 (1993)

LÜHKEN, G.; BUSCHMANN, A.; GROSCHUP, M.H.; ERHARD, G.:

Prion protein allele A136H154Q171 is associated with high susceptibility to scrapie in purebred and crossbred German Merinoland sheep. Arch. Virol. 149 (2004), 1571 - 1580

MCRAE, A.F.; MCEVAN, J.C.; DODDS, K.G.; WILSON, T.; CRAWFORD, A.M.; SLATE, J.: Linkage Disequilibrium in domestic sheep. Genetics 160 (2002) 1113-1122

PROKOPOVA, L.; LEWIS, R.M.; DINGWALL, W.S.; SIMM, G.;

Scrapie genotype: A correlation with lean growth rate? 7th World Congress on Genetics applied to livestock Production, Montpellier, 19.- 23. August, 31 2002, 779-782

TONGUE, S.C.; WILESMITH, J.W.; COOK, C.J.: 
Frequencies of prion protein (PrP) genotypes and distribution of ages in 15 scrapie - affected flocks in Great Britain. Vet. Rec. 154 (2004), 9 - 16

WALLING, G.A.; FISHER, P.M.; WILSON, A.D.; MCTEIR, B.L.; SIMM, G.; BISHOP, S.C.:

Mapping of quantitative trait loci for growth and carcass traits in commercial sheep populations. J. Anim. Sci. 82 (2004), 2234-2245

Eingegangen: 24.01.2005

Akzeptiert: 01.06.2005

Anschriften der Verfasser

Dr. ERHARD GERNAND*, Dr. HEIKE LENZ

Thüringer Landesanstalt für Landwirtschaft Jena, Abt. Tierproduktion

Standort Clausberg

Clausberg 7

99819 OBERELLEN

*Autor für Korrespondenz

E-mail: e.gernand@clausberg.tll.de

E-mail: h.lenz@clausberg.tll.de

Dr. UDO MOOG

Tiergesundheitsdienst Thüringen e.V.

Naumburger Str. 96b

03643 JENA

E-mail: udomoog@hotmail.com

Dr. RALF WAßMUTH

Thüringer Landesanstalt für Landwirtschaft Jena, Abt. Tierproduktion Naumburger Str. 98

07743 JENA

E-mail: r.wassmuth@jena.tll.de 